\title{
Uso da corrente russa na reabilitação neurológica de paciente com traumatismo crânio encefálico: relato de caso
}

\author{
Use of the Russian electrical stimulation in the neurological \\ rehabilitation of traumatic brain injury patient: case report \\ Arley Ribeiro de Castro ${ }^{1,2}$, Rita di Cássia de Oliveira Angelo ${ }^{1,3}$, Paulo Adriano Schwingel ${ }^{3}$ \\ ${ }^{1}$ Faculdade Inspirar (FI) - Petrolina (PE), Brasil. \\ ${ }^{2}$ Clínica +Fisio Pilates e Fisioterapia (+Fisio) - Petrolina (PE), Brasil. \\ ${ }^{3}$ Laboratório de Pesquisas em Desempenho Humano (LAPEDH), Universidade de Pernambuco (UPE) - Petrolina (PE), Brasil.
}

DOI: http://dx.doi.org/10.7322/abcshs.v42i2.1012

\begin{abstract}
RESUMO
Introdução: O traumatismo cranioencefálico (TCE) é qualquer agressão capaz de lesão anatômica ou comprometimento funcional do couro cabeludo, crânio, meninges ou encéfalo, que pode causar importantes perdas cognitivas e motoras. Os principais mecanismos de trauma são os acidentes automobilísticos, quedas e as agressões físicas e/ou lesões por arma de fogo. Este estudo objetivou relatar os efeitos de um protocolo de intervenção fisioterapêutica que inclui o uso da Corrente Russa (CR) na reabilitação de um paciente com TCE. Relato de caso: Paciente do sexo masculino, 26 anos, mecânico, etilista crônico, diagnóstico médico de TCE e hemiparesia esquerda desproporcionada com predominância no membro superior, dependente de cadeira de rodas. A avaliação fisioterapêutica foi constituída de aferição de sinais vitais, avaliação dos reflexos superficiais e tendinosos e do tônus muscular, goniometria e teste de força muscular específico. Após avaliação inicial foi aplicado um protocolo de intervenção fisioterapêutico constituído de exercícios terapêuticos e eletroestimulação com $\mathrm{CR}$, com frequência de duas sessões semanais de 50 minutos de duração, por um período de 16 semanas, seguidas de reavaliação. Conclusão: $O$ protocolo de intervenção aplicado contribuiu para a reabilitação funcional do paciente, verificado pelo ganho de amplitude de movimento das articulações do punho, cotovelo, tornozelo e joelho esquerdos; e aumento na graduação de força muscular dos flexores e extensores do punho e do cotovelo, flexores e extensores do joelho e tornozelo esquerdos. Além disso, a deambulação independente foi restituída, com retorno à atividade profissional.
\end{abstract}

Palavras-chave: fisioterapia; terapia por estimulação elétrica; lesões encefálicas traumáticas; reabilitação.

\begin{abstract}
Introduction: The traumatic brain injury (TBI) is any aggression capable of causing damage or functional impairment of the scalp, skull, meninges or brain, which can bring significant cognitive and motor losses. The main mechanisms of trauma are traffic accidents, falls and physical aggression and/or firearm injuries. This study aimed to report the effects of an intervention protocol physical therapy which includes the use of Russian Electrical Stimulation (RES) in the rehabilitation of a patient with TBI. Case report: Male patient, 26, mechanic, chronic alcoholic, with medical diagnosis of TBI and left hemiparesis disproportionate predominantly in the arm, dependent on a wheelchair. The physical therapy evaluation consisted of measuring vital signs, evaluation of surface and tendon reflexes and muscle tone, goniometry and specific muscle strength test. After the initial evaluation it was applied a physiotherapy intervention protocol consisting of therapeutic and electrical stimulation exercises with RES, with frequency of two weekly sessions of 50 minutes, for 16 weeks, followed by reevaluation. Conclusion: The applied intervention protocol contributed to the rehabilitation patient's functional, checked through the range of motion gain in the joints of the wrist, elbow, ankle and left knee; and increased muscle strength undergraduate flexor and extensors of the wrist and the elbow, flexors and extensors of the knee and left ankle. Furthermore, the independent ambulation was restored, returning to the professional activity.
\end{abstract}

Keywords: physical therapy specialty; electric stimulation therapy; brain injuries, traumatic; rehabilitation. 


\section{INTRODUÇÃO}

Traumatismo cranioencefálico (TCE) é qualquer agressão capaz de causar lesão anatômica ou comprometimento funcional do couro cabeludo, crânio, meninges e/ou encéfalo. Ocorre por lesão direta ao parênquima encefálico ou forças de impacto e inércia sobre o crânio e o encéfalo, ocasionando lesões de estruturas vasculares e neuronais que culminam em importantes perdas cognitivas e motoras ${ }^{1}$.

Os principais mecanismos de trauma são os acidentes automobilísticos, responsáveis por $45 \%$ dos casos de TCE. Nessa categoria também se enquadram atropelamentos, acidentes motociclísticos, ciclísticos ou envolvendo outros veículos não motorizados. Além disso, quedas são responsáveis por outros $31 \%$ dos TCE e agressões físicas e/ou lesões por arma de fogo originam $11 \%$ dos casos $^{2}$.

Normalmente o tratamento de pacientes diagnosticados com TCE inclui fisioterapia motora, composta por exercícios terapêuticos e eletrotermofototerapia ${ }^{1}$. De forma rotineira, utiliza-se a eletroestimulação funcional (EEF), por ser considerada eficaz no reaprendizado de algumas funções motoras comprometidas em pacientes neurológicos, uma vez que sua estimulação auxilia na contração do músculo escolhido. Não fosse essa estimulação, esses músculos não seriam ativados ou seriam ativados de forma incorreta, e suas respectivas funções responderiam de forma anormal ${ }^{3}$.

Dentre os tipos de correntes empregadas na EEF, a Corrente Russa (CR) é uma corrente sinusoidal alternada (bifásica) de $2.500 \mathrm{~Hz}$ que tem se mostrado efetiva no tratamento de distintas patologias, uma vez que aumenta o trofismo e pode restabelecer a força muscular, sobretudo de músculos que apresentam déficit pós-imobilização ou lesão neural ${ }^{4}$. Tais características apontam a CR como tratamento de eleição para pacientes com TCE. Apesar de a CR apresentar resultados significativos em pacientes neurológicos, estudos de análise da eficácia desse tipo de corrente na reabilitação neurológica de pacientes com TCE não foram reportados na literatura até o presente momento. Nesse contexto, este estudo objetivou relatar os efeitos de um protocolo de intervenção fisioterapêutica que inclui o uso da CR na reabilitação de um paciente vítima de TCE por projétil de arma de fogo (PAF). Espera-se que os achados aqui relatados sinalizem a importância do emprego dessa metodologia em pacientes neurológicos e contribuam para o fomento de novos estudos.

\section{MÉTODOS}

Trata-se de um estudo de caso (pesquisa comparativa de antes e depois) realizado no setor ambulatorial de Fisioterapia do Hospital Universitário de Petrolina (HU), na cidade de Petrolina, Pernambuco. O estudo foi aprovado pelo Comitê de Ética e Pesquisa da Universidade de Pernambuco (CEPUPE) sob CAAE: 0302.0.097.000-10. O participante recebeu informações sobre o estudo e assinou o termo de consentimento livre e esclarecido (TCLE).

\section{RELATO DO CASO}

Paciente do sexo masculino, 26 anos, mecânico, etilista crônico, não dependente químico, massa corporal total (MCT) de 79,6 kg, estatura de $1,77 \mathrm{~m}$, diagnóstico médico de traumatismo crânio encefálico, vítima de ferimento por arma de fogo, atingido na região temporoparietal direita há aproximadamente 30 dias. Foi admitido no HU, permanecendo 10 dias na Unidade de Terapia Intensiva (UTI) e 10 dias na enfermaria do hospital. Evoluiu com perda súbita de força dos membros superior esquerdo (MSE) e inferior esquerdo (MIE), com diagnóstico cinético-funcional de hemiparesia à esquerda com predominância no membro superior, ficando restrito à cadeira de rodas.

Inicialmente procedeu-se a avaliação fisioterapêutica constituída de aferição da pressão arterial (PA) e frequência cardíaca (FC), avaliação dos reflexos superficiais e tendinosos e do tônus muscular pela escala modificada de Ashworth ${ }^{5}$, goniometria do punho, cotovelo, tornozelo e joelho esquerdos, e teste de força muscular específico da musculatura flexora e extensora do punho, cotovelo, tornozelo e joelho esquerdos.

Após avaliação inicial, foi aplicado o protocolo de intervenção fisioterapêutico, com frequência de duas vezes por semana e duração de 50 minutos por sessão, durante 16 semanas, totalizando 32 atendimentos. Todos os componentes presentes na avaliação inicial foram reavaliados após as 16 semanas de aplicação do protocolo. Tanto as avaliações quanto os procedimentos foram realizados no setor ambulatorial de Fisioterapia do HU.

\section{Procedimentos}

O protocolo de intervenção fisioterapêutica aplicado neste estudo foi desenvolvido visando a otimizar a recuperação do paciente, e é apresentado a seguir:

- 10 minutos de alongamentos passivos com duas séries de 30 segundos cada, sendo 5 minutos para os músculos flexores e extensores do punho e cotovelo do MSE e 5 minutos para flexores e extensores do tornozelo e joelho do MIE;

- 15 minutos de aplicação de CR (frequência: $20 \mathrm{~Hz}$; largura de pulso: $300 \mu \mathrm{s}$; subida: $2 \mathrm{~s}$; on: $8 \mathrm{~s}$; descida: $2 \mathrm{~s}$; off: $24 \mathrm{~s}$; modo sincronizado $)^{6,7}$. Os eletrodos do MSE foram aplicados no ventre muscular do bíceps braquial e nos flexores do punho em uma sessão semanal, e nos tríceps braquial e extensores do punho na outra. No MIE, os eletrodos foram aplicados no ventre muscular do quadríceps femoral e do tibial anterior na primeira sessão semanal; e nos ventres musculares dos isquiotibiais e do gastrocnêmio na segunda sessão;

- 20 minutos de exercícios terapêuticos, sendo exercícios passivos de flexão e extensão do punho, cotovelo, tornozelo e joelho esquerdos, progredindo para exercícios ativos livres e depois resistidos. Para a execução dos exercícios resistidos, foram 
utilizados halteres (1 e $2 \mathrm{~kg}$ ), caneleiras (2,3 e 5,0 kg) e faixas elásticas (resistências média e forte), que foram empregados na medida em que o paciente aumentava o grau de força muscular nos respectivos movimentos; e

- 5 minutos de repouso ao final de cada sessão, com o paciente em sedestação, realizando exercícios respiratórios.

A avaliação e a reavaliação foram realizadas pelo mesmo examinador. Foram analisadas as características cineantropométricas, sendo a MCT aferida em quilogramas (kg) por meio de balança antropométrica mecânica (Welmy Indústria e Comércio Ltda., Santa Bárbara do Oeste, São Paulo, Brasil) e a estatura medida em metros $(\mathrm{m})$, pelo estadiômetro da própria balança. Obtevese o índice de massa corporal (IMC) através da divisão do valor da massa corporal total pelo valor da estatura ao quadrado $\left(\mathrm{IMC}=\mathrm{MCT} /\right.$ estatura $\left.^{2}\right)$.

A PA foi aferida com auxílio do esfigmomanômetro aneróide Premium com inflação e deflação manuais, e do estetoscópio do tipo Premium Rappaport (ambos da Accumed Produtos Médico-hospitalares Ltda., Duque de Caxias, Rio de Janeiro, Brasil). A FC foi obtida com auxílio da técnica palpatória da artéria radial, utilizando a contagem do número de batimentos em 1 minuto (Tabela 1 ).

O aparelho de CR utilizado foi o Neuromed 4080 Multi-corrent (Carci Indústria e Comércio de Aparelhos Cirúrgicos e Hospitalares Ltda., São Paulo, Brasil), com a manutenção em dia.

$\mathrm{Na}$ avaliação clínica, os sinais vitais (PA e FC) mantiveram valores dentro da normalidade em todas as sessões, não

Tabela 1: Características cineantropométricas do paciente

\begin{tabular}{|l|c|c|}
\hline Variáveis & Avaliação inicial & Avaliação final \\
\hline Massa corporal total $(\mathrm{kg})$ & 79,6 & 76,0 \\
\hline Estatura $(\mathrm{cm})$ & 177,0 & 177,0 \\
\hline IMC $\left(\mathrm{kg} / \mathrm{m}^{2}\right)$ & 25,4 & 24,3 \\
\hline PA sistólica $(\mathrm{mmHg})$ & 130 & 120 \\
\hline PA diastólica $(\mathrm{mmHg})$ & 80 & 80 \\
\hline FC (bpm) & 92 & 73 \\
\hline
\end{tabular}

IMC: índice de massa corporal; PA: pressão arterial; FC: frequência cardíaca. havendo intercorrência durante aplicação do protocolo, sem necessidade de interrupção. Em relação às características antropométricas, verificou-se que o paciente perdeu $3,6 \mathrm{~kg}$ de MCT após a intervenção fisioterapêutica, bem como saiu da faixa considerada sobrepeso segundo o IMC para a faixa de peso normal (Tabela 1).

$\mathrm{Na}$ avaliação fisioterapêutica inicial do hemicorpo esquerdo, foi observado que todos os reflexos superficiais e tendinosos dos MSE e MIE estavam presentes. O tônus muscular foi classificado como sendo hipertônico, grau 2 de acordo com a escala modificada de Ashworth ${ }^{5}$. No teste muscular manual (TMM) ${ }^{8}$, o paciente apresentou grau 0 para os músculos flexores e extensores do punho e cotovelo esquerdos, e grau 1 para os músculos flexores e extensores do tornozelo e joelho esquerdos.

A avaliação goniométrica inicial foi realizada de forma passiva, utilizando um goniômetro clínico (Sma Fisioterapia Produtos e Acessórios Médico-hospitalares Ltda., Salvador, Bahia, Brasil). Foram verificados os seguintes ângulos nas articulações do MSE: $40^{\circ}$ de extensão e $90^{\circ}$ de flexão do punho, e $0^{\circ}$ de extensão e $120^{\circ}$ de flexão do cotovelo. No MIE, observou-se: $15^{\circ}$ de flexão plantar e $15^{\circ}$ de dorsiflexão do tornozelo, e $0^{\circ}$ de extensão e $110^{\circ}$ de flexão do joelho (Tabela 2).

\section{RESULTADOS}

Nas primeiras quatro semanas de utilização do protocolo, foi verificado aumento na graduação da força dos grupamentos musculares avaliados do MIE, sendo possível a progressão de exercícios passivos para ativos-assistidos, com o paciente ainda dependente da cadeira de rodas. Não foi observada mudança na graduação da força dos músculos avaliados no MSE.

Com oito semanas, foi constatado incremento na graduação da força dos grupamentos musculares avaliados do MSE, sendo possível progredir de exercícios passivos para ativos-assistidos. No MIE, foram inseridos exercícios ativos e resistidos com caneleira de $2 \mathrm{~kg}$ para os movimentos de flexão e extensão do joelho em cadeia cinética aberta (CCA); e faixa elástica de resistência

Tabela 2: Avaliação de força muscular e goniometria antes a após a aplicação do protocolo

\begin{tabular}{|c|c|c|c|c|c|}
\hline \multicolumn{3}{|c|}{ Membro superior esquerdo } & \multicolumn{3}{|c|}{ Membro inferior esquerdo } \\
\hline Articulação & Inicial & Final & Articulação & Inicial & Final \\
\hline \multicolumn{6}{|c|}{ Avaliação da força muscular } \\
\hline Extensão de cotovelo & Grau 0 & Grau 2 & Extensão de joelho & Grau 1 & Grau 5 \\
\hline Flexão de cotovelo & Grau 0 & Grau 4 & Flexão de joelho & Grau 1 & Grau 5 \\
\hline Extensão de punho & Grau 0 & Grau 2 & Flexão plantar & Grau 1 & Grau 5 \\
\hline Flexão de punho & Grau 0 & Grau 4 & Dorsiflexão & Grau 1 & Grau 5 \\
\hline \multicolumn{6}{|l|}{ Avaliação goniométrica } \\
\hline Extensão de cotovelo & $0^{\circ *}$ & $0^{\circ}$ & Extensão de joelho & $0^{\circ *}$ & $0^{\circ}$ \\
\hline Flexão de cotovelo & $120^{\circ *}$ & $120^{\circ}$ & Flexão de joelho & $110^{\circ *}$ & $100^{\circ}$ \\
\hline Extensão de punho & $40^{\circ *}$ & $49^{\circ * \star}$ & Flexão plantar & $15^{\circ *}$ & $15^{\circ}$ \\
\hline Flexão de punho & $90^{\circ *}$ & $70^{\circ}$ & Dorsiflexão & $15^{\circ *}$ & $14^{\circ}$ \\
\hline
\end{tabular}

*Valores obtidos com movimentação passiva; **Valor obtido com movimentação ativa/assistida. 
média para dorsiflexão e flexão plantar do tornozelo, sendo possível treinar deambulação de curta distância com apoio bilateral nas barras paralelas (Tabela 3).

Após 12 semanas, foram inseridos exercícios ativos e resistidos com halter de $1 \mathrm{~kg}$ para os movimentos de flexão do punho e do cotovelo esquerdos. No MIE, foram mantidos os exercícios resistidos, com aumento da carga para $3 \mathrm{~kg}$ nos movimentos de flexão e extensão de joelho, e faixa elástica de resistência forte para dorsiflexão e flexão plantar de tornozelo. O paciente ficou independente da cadeira de rodas, sendo possível realizar a marcha com auxílio de um par de muletas canadenses em marcha de 4 pontos.

Em seguida, com 16 semanas de protocolo, foram mantidos os exercícios resistidos para flexão do punho e do cotovelo esquerdos, com aumento da carga para $2 \mathrm{~kg}$; além de exercícios ativos para extensão do punho e do cotovelo no mesmo lado. O paciente alcançou graduação 4 e 2 de força muscular no MSE, respectivamente, para os flexores e extensores do punho e do cotovelo. Para o MIE, permaneceram os exercícios resistidos com aumento da carga para $5 \mathrm{~kg}$ nos movimentos de flexão e extensão do joelho, e faixa elástica de resistência forte para dorsiflexão e flexão plantar de tornozelo. O paciente atingiu a graduação 5 de força muscular para todos os movimentos avaliados, além de deambular com o apoio de apenas uma muleta canadense, conseguindo retornar à sua atividade profissional (Tabela 4).

Na reavaliação goniométrica, observa-se a recuperação da amplitude de movimento, sendo possível obter os valores dos ângulos das articulações avaliadas de forma ativa, já que o paciente conseguiu realizar todos os movimentos ativamente. $\mathrm{O}$ tônus muscular foi classificado como normal, recebendo pontuação 0 de acordo com a escala modificada de Ashworth ${ }^{5}$.

Tabela 3: Progressão do paciente nos exercícios da primeira à oitava semana

\begin{tabular}{|c|c|c|c|c|c|c|c|c|}
\hline Articulação & 1 sem. & 2 sem. & 3 sem. & 4 sem. & 5 sem. & $6 \mathrm{sem}$. & 7 sem. & 8 sem. \\
\hline \multicolumn{9}{|c|}{ Membro superior esquerdo } \\
\hline $\begin{array}{l}\text { Extensão } \\
\text { de cotovelo }\end{array}$ & Passivo & Passivo & Passivo & Passivo & Passivo & $\begin{array}{c}\text { Ativo/ } \\
\text { Assistido }\end{array}$ & $\begin{array}{c}\text { Ativo/ } \\
\text { Assistido }\end{array}$ & $\begin{array}{c}\text { Ativo/ } \\
\text { Assistido }\end{array}$ \\
\hline $\begin{array}{l}\text { Flexão de } \\
\text { cotovelo }\end{array}$ & Passivo & Passivo & Passivo & Passivo & Passivo & $\begin{array}{c}\text { Ativo/ } \\
\text { Assistido }\end{array}$ & $\begin{array}{c}\text { Ativo/ } \\
\text { Assistido }\end{array}$ & $\begin{array}{c}\text { Ativo/ } \\
\text { Assistido }\end{array}$ \\
\hline $\begin{array}{l}\text { Extensão } \\
\text { de punho }\end{array}$ & Passivo & Passivo & Passivo & Passivo & Passivo & $\begin{array}{c}\text { Ativo/ } \\
\text { Assistido }\end{array}$ & $\begin{array}{c}\text { Ativo/ } \\
\text { Assistido }\end{array}$ & $\begin{array}{c}\text { Ativo/ } \\
\text { Assistido }\end{array}$ \\
\hline $\begin{array}{l}\text { Flexão de } \\
\text { punho }\end{array}$ & Passivo & Passivo & Passivo & Passivo & Passivo & $\begin{array}{c}\text { Ativo/ } \\
\text { Assistido }\end{array}$ & $\begin{array}{c}\text { Ativo/ } \\
\text { Assistido }\end{array}$ & $\begin{array}{c}\text { Ativo/ } \\
\text { Assistido }\end{array}$ \\
\hline \multicolumn{9}{|c|}{ Membro inferior esquerdo } \\
\hline $\begin{array}{l}\text { Extensão } \\
\text { de joelho }\end{array}$ & Passivo & Passivo & $\begin{array}{c}\text { Ativo/ } \\
\text { Assistido }\end{array}$ & $\begin{array}{c}\text { Ativo/ } \\
\text { Assistido }\end{array}$ & Ativo & Ativo & $\begin{array}{l}\text { Resistido } \\
\text { (2 kg) }\end{array}$ & $\begin{array}{l}\text { Resistido } \\
\text { (2 kg) }\end{array}$ \\
\hline $\begin{array}{l}\text { Flexão de } \\
\text { joelho }\end{array}$ & Passivo & Passivo & $\begin{array}{c}\text { Ativo/ } \\
\text { Assistido }\end{array}$ & $\begin{array}{c}\text { Ativo/ } \\
\text { Assistido }\end{array}$ & Ativo & Ativo & $\begin{array}{c}\text { Resistido } \\
(2 \mathrm{~kg})\end{array}$ & $\begin{array}{l}\text { Resistido } \\
\text { (2 kg) }\end{array}$ \\
\hline $\begin{array}{l}\text { Flexão } \\
\text { plantar }\end{array}$ & Passivo & Passivo & $\begin{array}{c}\text { Ativo/ } \\
\text { Assistido }\end{array}$ & $\begin{array}{c}\text { Ativo/ } \\
\text { Assistido }\end{array}$ & Ativo & Ativo & $\begin{array}{c}\text { Faixa Elástica } \\
\text { (RM) }\end{array}$ & $\begin{array}{c}\text { Faixa Elástica } \\
(\mathrm{RM})\end{array}$ \\
\hline Dorsiflexão & Passivo & Passivo & $\begin{array}{c}\text { Ativo/ } \\
\text { Assistido }\end{array}$ & $\begin{array}{c}\text { Ativo/ } \\
\text { Assistido }\end{array}$ & Ativo & Ativo & $\begin{array}{c}\text { Faixa Elástica } \\
\text { (RM) }\end{array}$ & $\begin{array}{c}\text { Faixa Elástica } \\
(\mathrm{RM})\end{array}$ \\
\hline
\end{tabular}

RM: resistência média; sem.: semana(s).

Tabela 4: Progressão do paciente nos exercícios da nona à décima sexta semana

\begin{tabular}{|c|c|c|c|c|c|c|c|c|}
\hline Articulação & 9 sem. & 10 sem. & 11 sem. & 12 sem. & 13 sem. & 14 sem. & 15 sem. & 16 sem. \\
\hline \multicolumn{9}{|c|}{ Membro superior esquerdo } \\
\hline $\begin{array}{l}\text { Extensão } \\
\text { de cotovelo }\end{array}$ & $\begin{array}{c}\text { Ativo/ } \\
\text { Assistido }\end{array}$ & $\begin{array}{c}\text { Ativo/ } \\
\text { Assistido }\end{array}$ & $\begin{array}{c}\text { Ativo/ } \\
\text { Assistido }\end{array}$ & $\begin{array}{c}\text { Ativo/ } \\
\text { Assistido }\end{array}$ & $\begin{array}{c}\text { Ativo/ } \\
\text { Assistido }\end{array}$ & $\begin{array}{c}\text { Ativo/ } \\
\text { Assistido }\end{array}$ & $\begin{array}{c}\text { Ativo/ } \\
\text { Assistido }\end{array}$ & $\begin{array}{c}\text { Ativo/ } \\
\text { Assistido }\end{array}$ \\
\hline $\begin{array}{l}\text { Flexão de } \\
\text { cotovelo }\end{array}$ & Ativo & Ativo & Ativo & $\begin{array}{l}\text { Resistido } \\
\text { (1 kg) }\end{array}$ & $\begin{array}{l}\text { Resistido } \\
\text { (1 kg) }\end{array}$ & $\begin{array}{l}\text { Resistido } \\
\text { (1 kg) }\end{array}$ & $\begin{array}{l}\text { Resistido } \\
\text { (2 kg) }\end{array}$ & $\begin{array}{c}\text { Resistido } \\
(2 \mathrm{~kg})\end{array}$ \\
\hline $\begin{array}{l}\text { Extensão } \\
\text { de punho }\end{array}$ & $\begin{array}{c}\text { Ativo/ } \\
\text { Assistido }\end{array}$ & $\begin{array}{c}\text { Ativo/ } \\
\text { Assistido }\end{array}$ & $\begin{array}{c}\text { Ativo/ } \\
\text { Assistido }\end{array}$ & $\begin{array}{c}\text { Ativo/ } \\
\text { Assistido }\end{array}$ & $\begin{array}{c}\text { Ativo/ } \\
\text { Assistido }\end{array}$ & $\begin{array}{c}\text { Ativo/ } \\
\text { Assistido }\end{array}$ & $\begin{array}{c}\text { Ativo/ } \\
\text { Assistido }\end{array}$ & $\begin{array}{c}\text { Ativo/ } \\
\text { Assistido }\end{array}$ \\
\hline $\begin{array}{l}\text { Flexão de } \\
\text { punho }\end{array}$ & Ativo & Ativo & Ativo & $\begin{array}{l}\text { Resistido } \\
\text { (1 kg) }\end{array}$ & $\begin{array}{l}\text { Resistido } \\
\text { (1 kg) }\end{array}$ & $\begin{array}{l}\text { Resistido } \\
\text { (1 kg) }\end{array}$ & $\begin{array}{l}\text { Resistido } \\
(2 \mathrm{~kg})\end{array}$ & $\begin{array}{l}\text { Resistido } \\
\text { (2 kg) }\end{array}$ \\
\hline \multicolumn{9}{|c|}{ Membro inferior esquerdo } \\
\hline $\begin{array}{l}\text { Extensão } \\
\text { de joelho }\end{array}$ & $\begin{array}{l}\text { Resistido } \\
(2 \mathrm{~kg})\end{array}$ & $\begin{array}{l}\text { Resistido } \\
\text { (3 kg) }\end{array}$ & $\begin{array}{l}\text { Resistido } \\
\text { (3 kg) }\end{array}$ & $\begin{array}{l}\text { Resistido } \\
\text { (3 kg) }\end{array}$ & $\begin{array}{l}\text { Resistido } \\
\text { (3 kg) }\end{array}$ & $\begin{array}{l}\text { Resistido } \\
\text { (5 kg) }\end{array}$ & $\begin{array}{l}\text { Resistido } \\
\text { (5 kg) }\end{array}$ & $\begin{array}{l}\text { Resistido } \\
\text { (5 kg) }\end{array}$ \\
\hline $\begin{array}{l}\text { Flexão de } \\
\text { joelho }\end{array}$ & $\begin{array}{l}\text { Resistido } \\
\text { (2 kg) }\end{array}$ & $\begin{array}{l}\text { Resistido } \\
\text { (3 kg) }\end{array}$ & $\begin{array}{l}\text { Resistido } \\
\text { (3 kg) }\end{array}$ & $\begin{array}{l}\text { Resistido } \\
\text { (3 kg) }\end{array}$ & $\begin{array}{l}\text { Resistido } \\
\text { (3 kg) }\end{array}$ & $\begin{array}{l}\text { Resistido } \\
\text { (5 kg) }\end{array}$ & $\begin{array}{l}\text { Resistido } \\
\text { (5 kg) }\end{array}$ & $\begin{array}{l}\text { Resistido } \\
\text { (5 kg) }\end{array}$ \\
\hline $\begin{array}{l}\text { Flexão } \\
\text { plantar }\end{array}$ & $\begin{array}{c}\text { Faixa Elástica } \\
\text { (RM) }\end{array}$ & $\begin{array}{c}\text { Faixa Elástica } \\
\text { (RM) }\end{array}$ & $\begin{array}{c}\text { Faixa Elástica } \\
\text { (RM) }\end{array}$ & $\begin{array}{c}\text { Faixa Elástica } \\
(\mathrm{RF})\end{array}$ & $\begin{array}{c}\text { Faixa Elástica } \\
(\mathrm{RF})\end{array}$ & $\begin{array}{c}\text { Faixa Elástica } \\
(\mathrm{RF})\end{array}$ & $\begin{array}{c}\text { Faixa Elástica } \\
(\mathrm{RF})\end{array}$ & $\begin{array}{c}\text { Faixa Elástica } \\
\text { (RF) }\end{array}$ \\
\hline Dorsiflexão & $\begin{array}{c}\text { Faixa Elástica } \\
\text { (RM) }\end{array}$ & $\begin{array}{c}\text { Faixa Elástica } \\
\text { (RM) }\end{array}$ & $\begin{array}{c}\text { Faixa Elástica } \\
\text { (RM) }\end{array}$ & $\begin{array}{c}\text { Faixa Elástica } \\
(\mathrm{RF})\end{array}$ & $\begin{array}{c}\text { Faixa Elástica } \\
(\mathrm{RF})\end{array}$ & $\begin{array}{c}\text { Faixa Elástica } \\
(\mathrm{RF})\end{array}$ & $\begin{array}{c}\text { Faixa Elástica } \\
(\mathrm{RF})\end{array}$ & $\begin{array}{c}\text { Faixa Elástica } \\
\text { (RF) }\end{array}$ \\
\hline
\end{tabular}

RM: resistência média; RF: resistência forte; sem.: semana(s). 


\section{DISCUSSÃO}

Este relato de caso demonstrou os benefícios da intervenção da fisioterapia utilizando a CR sobre parâmetros antropométricos, neurológicos, musculares e funcionais.

Segundo Sousa e Koizumi ${ }^{9}$, o tempo de recuperação total do trauma de pacientes vítimas de TCE varia entre 6 e 12 meses, independentemente da gravidade; esse é o período o tempo necessário para que o paciente retorne ao mesmo estado funcional anterior ao do trauma e às suas atividades profissionais. Quando relacionamos os resultados obtidos, observa-se que o tempo de recuperação foi menor, com o paciente recuperando sua funcionalidade e retornando à sua atividade profissional dois meses antes do tempo mínimo estabelecido no estudo anterior?. O resultado aqui encontrado nos permite supor que o protocolo fisioterapêutico aplicado, i.e., com uso da CR, contribuiu para a otimização da reabilitação do paciente em questão.

De acordo com Salvini et al. ${ }^{10}$, a eletroestimulação possui várias indicações terapêuticas, dentre elas reparo tissular, analgesia, eletrodiagnóstico, treino funcional e de força em pacientes neurológicos, além de treino de força muscular em indivíduos saudáveis. Cunha et al. ${ }^{11}$ também afirmam que há efeito positivo na aplicação da eletroestimulação, apresentando importante ação terapêutica e prevenindo contraturas musculares. Além disso, a estimulação neuromuscular diminui a espasticidade e aumenta a força muscular, facilitando a recuperação motora do membro acometido ${ }^{10,11}$.

Dentre os diferentes tipos de correntes elétricas disponíveis (bai$\mathrm{xa}$, média e alta frequência), a CR foi escolhida para esse paciente por ser considerada uma modalidade de eletroestimulação de média frequência com alta tolerabilidade e a mais eficaz para a produção de força comparada a uma modalidade de baixa frequência, bem como é indicada para modulação da espasticidade ${ }^{12-14}$.

A utilização de $20 \mathrm{~Hz}$ tem sido associada a maior eficiência nos padrões de recrutamento neural, uma vez que consegue recrutar unidades motoras de tamanho médio (fibras mistas) que geram tanto força quanto hipertrofia dos músculos esqueléticos. Por fim, a largura de pulso foi estabelecida pelo tamanho das fibras e os tempos de contração e repouso foram determinados visando ao recrutamento muscular significativo sem fadiga muscular. Esses parâmetros seguiram dados encontrados na literatura, respeitando o tipo de fibra muscular (mista), a intensidade tolerada pelo paciente, e o tempo de terapia para evitar a fadiga ${ }^{6,7}$.

O diferencial do presente estudo encontra-se na proposição de aplicar a CR como recurso terapêutico em pacientes com TCE. Artioli et al. ${ }^{15}$ defendem a utilização da CR, visto que a mesma proporciona manutenção das propriedades histológicas do músculo, evitando atrofia musculoesquelética por denervação. No entanto, essa contração muscular induzida ocorre de maneira diferente da contração fisiológica, com recrutamento inicial de fibras do tipo II (rápidas) seguido das do tipo I (lentas). Isso, embora produza maior força muscular, pode acarretar em uma fadiga mais rápida caso não seja respeitado o tempo de repolarização nervosa, ou seja, o descanso da fibra muscular ${ }^{12,15}$.

\section{CONSIDERAÇÕES FINAIS}

Os resultados obtidos demonstram que, após o tratamento fisioterapêutico, houve restabelecimento do quadro clínico e funcional, o que pôde ser claramente observado no retorno do paciente às atividades diárias e laborais.

Contudo, em função das restrições metodológicas implicadas no relato de um único caso, é necessário que sejam fomentados mais estudos com delineamento experimental e que analisem de forma controlada os efeitos da CR em pacientes com TCE, no sentido de subsidiar a prática clínica do fisioterapeuta baseada em evidências.

\section{REFERÊNCIAS}

1. Almeida TLT, Falkenburg L, Nascimento RZR, Reis CA, Sales VC, Pedroso TD, et al. Traumatic brain injury: rehabilitation. Acta Fisiatr. 2012;19(2):130-7

http://dx.doi.org/10.5935/0104-7795.20120020

2. Dal Secchi MM, Moraes JFS, Castro FB. Fratura de osso temporal em pacientes com traumatismo crânio-encefálico. Arq Int Otorrinolaringol. 2012;16(1):62-6. http://dx.doi.org/10.7162/S1809-48722012000100009

3. Corrêa FI, Corrêa JCF, Tessarolo AA, Melo AS, Sampaio LMM, Costa MS, et al. Avaliação do ácido lático em indivíduos com hemiparesia pós-acidente vascular encefálico após estimulação elétrica para fortalecimento muscular. Fisioter Pesq. 2009;16(2):178-82.

http://dx.doi.org/10.1590/S1809-29502009000200015

4. AbdallaDR, Bertoncello D, Carvalho LC. Avaliação das propriedades mecânicas do músculo gastrocnêmio de ratas imobilizado e submetido à corrente russa. Fisioter Pesq. 2009;16(1):59-64. http://dx.doi.org/10.1590/S1809-29502009000100011
5. Teive HAG, Zonta M, Kumagai Y. Tratamento da espasticidade: uma atualização. Arq Neuro-Psiquiatr. 1998;56(4):852-8. http://dx.doi.org/10.1590/s0004-282x1998000500025

6. Avila MA, Brasileiro JS, Salvini TF. Electrical stimulation and isokinetic training: effects on strength and neuromuscular properties of healthy young adults. Rev Bras Fisioter. 2008;12(6):435-40. http://dx.doi.org/10.1590/S1413-35552008005000006

7. Briel AF, Massoni J. Efeito da eletroestimulação por corrente russa e exercício de fortalecimento da força e trofismo muscular do quadriceps femoral não dominante. Rev Biol Saúde Unisep. 2009;3(1):34-45.

8. Bohannon RW. Manual muscle testing: does it meets the standards of an adequate screening test? Clin Rehabil. 2005;19(6):662-7. http://dx.doi.org/10.1191/0269215505cr873oa

9. Sousa RMC, Koizumi MS. Recuperação das vítimas de traumatismo crânio-encefálico no período de 1 ano após o trauma. Rev Esc Enf USP. 1996;30(3):484-500 http://dx.doi.org/10.1590/S0080-62341996000300010 
10. Salvini TF, Durigan JLQ, Peviani SM, Russo TL. Efeitos da eletroestimulação e do alongamento muscular sobre a adaptação do músculo desnervado - implicações para a fisioterapia. Rev Bras Fisioter. 2012;16(3):175-83.

http://dx.doi.org/10.1590/S1413-35552012005000027

11. Cunha AS, de Freitas Junior JHA, Lopes Junior JEG, Figueiredo ADJ. Intervenção da fisioterapia na lesão do plexo braquial através de FES e cinesioterapia. Rev Fisioter Saúde Func. 2013;2(1):62-8.

12. Meireles ALF, Meireles LCF, Queiroz JCES, Tassitano RM, Soares FO, de Oliveira AS. Eficácia da eletroestimulação muscular expiratória na tosse de pacientes após acidente vascular encefálico. Fisioter Pesqui. 2012;19(4):314-9.

http://dx.doi.org/10.1590/S1809-29502012000400004
13. Ward AR, Shkuratova N. Russian electrical stimulation: the early experiments. Phys Ther. 2002;82(10):1019-30.

http://dx.doi.org/10.1093/ptj/82.10.1019

14. Leung J, Harvey LA, Moseley AM, Whiteside B, Simpson M, Stroud K. Standing with electrical stimulation and splinting is no better than standing alone for management of ankle plantarflexion contractures in people with traumatic brain injury: a randomised trial. J Physiother. 2014;60(4):201-8.

http://dx.doi.org/10.1016/j.jphys.2014.09.007

15. Artioli DP, do Nascimento ESP, dos Santos JC, Celeste LFN, Santini $\mathrm{L}$, de Andrade Junior MC, et al. O uso da corrente polarizada na fisioterapia. Rev Bras Clin Med. 2011;9(6):428-31. 